\title{
OFERTA Y DEMANDA DE MÉDICOS ESPECIALISTAS EN LOS ESTABLECIMIENTOS DE SALUD DEL MINISTERIO DE SALUD: BRECHAS A NIVEL NACIONAL, POR REGIONES Y TIPO DE ESPECIALIDAD
}

\author{
Leslie Zevallos ${ }^{1, a}$, Reyna Pastor $2, b$, Betsy Moscoso $2, a$
}

\begin{abstract}
RESUMEN
Objetivos. Caracterizar la oferta, la demanda y la brecha de médicos especialistas en establecimientos del Ministerio de Salud del Perú a nivel nacional, regional y por tipo de especialidad. Materiales y métodos. Estudio descriptivo, observacional a través del cual se calculó la oferta de médicos especialistas utilizando fuentes secundarias del MINSA. El análisis de la demanda de médicos especialistas se basó en dos metodologías: necesidades de especialistas según la norma de categorización de los establecimientos de salud y según el perfil epidemiológico y demográfico. La diferencia aritmética entre la demanda calculada y la oferta fue el procedimiento utilizado para calcular la brecha de médicos especialistas. Resultados. EI MINSA, a nivel nacional, tiene una oferta total de 6074 médicos especialistas de los cuales el $61,5 \%$ pertenecen a las especialidades clínicas, 33,2\% a especialidades quirúrgicas, $4,9 \%$ a especialidades de apoyo al diagnóstico y tratamiento y $0,4 \%$ a las especialidades de salud pública. Según la norma de categorización existe una demanda total de 11176 médicos especialistas y según el perfil epidemiológico y demográfico, de 11738 . Las brechas estimadas a nivel nacional son similares en ambos métodos, aunque difieren ampliamente en las regiones y por tipo de especialidad. A nivel de región, las brechas son mayores en Loreto, Piura, Puno y Madre de Dios cuando se usa la valoración del déficit en forma relativa a la oferta. En cuanto a especialidad, la brecha es mayor en las cuatro especialidades básicas: ginecología-obstetricia, pediatría, medicina interna y cirugía general. Conclusiones. Existe una gran brecha entre la oferta y la demanda de médicos especialistas a nivel nacional y regional que, de forma agregada, representa aproximadamente el $45 \%$ de la oferta actual, independientemente del método de estimación empleado.
\end{abstract}

Palabras clave: Médicos; Recursos humanos; Equidad; Política de salud (fuente: DeCS BIREME).

\section{SUPPLY AND DEMAND OF MEDICAL SPECIALISTS IN THE HEALTH FACILITIES OF THE MINISTRY OF HEALTH: NATIONAL, REGIONAL AND BY TYPE OF SPECIALTY GAPS}

\begin{abstract}
Objectives. To characterize the supply, demand and the gap of medical specialists in facilities of the Ministry of Health of Peru (MINSA) at the national, regional and specialty type levels. Materials and methods. Observational, descriptive study through which we calculated the supply of medical specialists using secondary sources of MINSA. The analysis of the demand for medical specialists was based on two methodologies: the need for specialists according to the guidelines of classification of the health facilities and according to the epidemiological and demographic profile. The arithmetic difference between the estimated demand and the supply was the procedure used to calculate the gap of medical specialists. Results. The Ministry of Health has a total supply at the national level of 6,074 medical specialists of which $61.5 \%$ belong to the clinical specialties, $33.2 \%$ to the surgical specialties, $4.9 \%$ specialities related to aid to diagnosis and treatment and $0.4 \%$ to public health specialties. According to the categorization guideline there is a total demand of 11,176 medical specialists and according to the epidemiological and demographic profile of 11,738. The national estimated gaps found are similar in both methods, although they differ widely across regions and by type of specialty. At the regional level, the gaps are greater in Loreto, Piura, Puno and Madre de Dios when estimating the defficit in relation to the supply. Regarding the speciality, the gap is greater in the four basic specialties: gynecology and obstetrics, pediatrics, internal medicine and general surgery. Conclusions. There is a waid gap between supply and demand of medical specialists at the national and regional levels, as a whole representing approximately $45 \%$ of the current offer, regardless of the estimation method.
\end{abstract}

Key words: Physicians; Human esources; Equity; Health policy (source: MeSH NLM).

Facultad de Medicina, Universidad Científica del Sur. Lima, Perú.

2 Observatorio de Recursos Humanos, Dirección General de Gestión del Desarrollo de los Recursos Humanos, Ministerio de Salud. Lima, Perú.

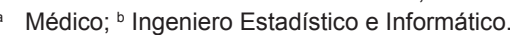

Recibido: 26-04-11 Aprobado: 01-06-11 


\section{INTRODUCCIÓN}

El objetivo de todo sistema de salud es preservar y mejorar la salud de la población y, para ello, debe cumplir las siguientes funciones: rectoría, financiamiento, provisión de servicios y creación de recursos humanos y físicos ${ }^{(1)}$. Esta última función hace referencia a los insumos básicos que se requieren para la producción de servicios, especialmente los recursos humanos, la infraestructura y el equipamiento. El Estado es el principal responsable del adecuado desempeño del sistema de salud y en el Perú es el Ministerio de Salud (MINSA) el responsable de ello.

El Perú posee un sistema de salud segmentado, por cuanto las funciones señaladas previamente son desarrolladas de manera autónoma y poco articulada por los subsectores público y privado. Todo esto se vuelve más complejo al identificar que dentro del subsector público, existen varios prestadores de servicios de salud que se financian por diferentes agentes y realizan la función de prestación de manera poco coordinada.

El principal agente financiador de los servicios de salud lo constituyen los hogares (gasto de bolsillo), que representan el $34,2 \%$, seguido por el gobierno con $30,7 \%$, los empleadores representan el $30,5 \%$ y el $4,6 \%$ restante por cooperantes externos, donantes internos y otros. El financiamiento de los establecimientos de salud por parte del gobierno se canaliza por intermedio de diferentes entidades: para los establecimientos de salud del MINSA su financiamiento proviene del presupuesto asignado al MINSA y Regiones, para los establecimientos de salud de EsSalud en cambio proviene del presupuesto asignado al Ministerio de Trabajo y para los establecimientos de salud de la Sanidad de las Fuerzas Armadas y Policía Nacional proviene de los Ministerios de Defensa y del Interior (2).

En cuanto a cobertura de seguros de salud el $64,7 \%$ de la población del país se encuentra afiliada a algún seguro de salud; de los cuales el 39,3\% accede en mayor proporción al Seguro Integral de Salud, seguido de aquellos que acceden al Seguro Social EsSalud $(20,7 \%)$ y a otros seguros $(4,67 \%)$ que incluyen seguro privado de salud, seguro de las Fuerzas Armadas y Policiales, entre otros ${ }^{(3)}$.

En los últimos años, en el sector salud se han registrado diversas situaciones con repercusión en el incremento de la demanda de servicios de salud por parte de la población. En primer lugar, tenemos los cambios en la dinámica demográfica ${ }^{(4)}$, que ha generado el cambio de las necesidades y demandas de la población. En segundo lugar, el acceso de la población al aseguramiento universal en salud (5). En tercer lugar, tenemos la fuerte inversión que se ha venido desarrollando en infraestructura y equipos ${ }^{(5)}$, reflejado esto en construcción de nuevos establecimientos de salud o ampliación y mejoramiento de la capacidad resolutiva de los ya existentes.

No obstante, la situación de los recursos humanos en salud no ha caminado a la par con estos cambios; si nos referimos solo al recurso humano médico especialista, contradictoriamente se observa que las plazas para la formación especializada (residentado) no han sufrido la misma variación con respecto al crecimiento y demanda de la población. Asimismo, la inversión que se viene realizando en hospitales no va de la mano con una política de incentivos que haga más atractiva las plazas para el médico especialista, esto se agrava al observar la oleada de inmigración de médicos que se viene registrando en los últimos años ${ }^{(6)}$.

Es por ello que determinar la oferta y demanda de servicios de salud y, en este caso de forma más específica las referidas a médicos especialistas, siempre es objeto de atención ${ }^{(7-9)}$. Entre los métodos empleados para planificar recursos humanos en salud tenemos los que se basan en: a) la oferta, en la cual se estima el personal en el momento base y luego se realizan proyecciones; b) la demanda o utilización, en la cual se intenta confrontar la necesidad de médicos especialistas para satisfacer la demanda existente; c) la carencia, en la cual se intenta satisfacer las necesidades de atención de salud en términos de tasas de crecimiento de la población y d) benchmarking, con el cual se busca estándares o parámetros a imitar. En el Perú existen diferentes contribuciones e investigaciones previas relacionadas con el tema ${ }^{(6,10-12)}$. En todos los casos, el objetivo común de estos estudios ha sido proporcionar información concreta para la toma de decisiones y planificación en salud desde diversas perspectivas y metodologías, y en todos los casos el hallazgo central es repetitivo: en el marco de un entorno carente de gobernabilidad y regulación de formación y distribución de profesionales de la salud, entre ellos los médicos y especialistas o subespecialistas, determinado por un modelo de provisión de servicios no articulado, el gran perdedor es el potencial usuario de servicios preventivos, curativos o recuperativos en especial aquellos con mayor vulnerabilidad como son los pobres. Sin embargo, estos estudios previos, que documentan ampliamente la realidad del quehacer médico, son de tipo agregado a nivel nacional (por territorio o especialidad), solo exploran algunas regiones o subregiones y se limitan al uso de una sola metodología de valoración de las brechas cuando estas han sido valoradas. 
En consecuencia, existe la motivación y necesidad general, de identificar la necesidad de médicos especialistas para el MINSA y las direcciones regionales de salud, y confrontar estos hallazgos con rigurosidad desde más de un frente metodológico ${ }^{(13)}$, con la finalidad de contar con insumos para la planificación de recursos humanos de salud.

El objetivo principal de este estudio fue caracterizar la oferta y la demanda de especialistas de los establecimientos del MINSA y determinar la brecha (o necesidad por cobertura inadecuada) a nivel nacional, regional y por tipo genérico y específico de especialidad médica.

\section{MATERIALES Y MÉTODOS}

\section{TIPO DE ESTUDIO}

Se realizó un estudio observacional descriptivo de tipo transversal dirigido a valorar la oferta, la demanda y la brecha de médicos especialistas a nivel nacional y por regiones. Fue ejecutado durante el año 2010 y auspiciado por la Dirección General de Gestión del Desarrollo de Recursos Humanos del MINSA a través del Observatorio de Recursos Humanos en Salud, bajo el nombre de "Recopilación, Procesamiento y Análisis de Información sobre la Necesidad de Médicos Especialistas para los Establecimientos de Salud del MINSA y las Regiones del Perú", el estudio contó con autorización para su publicación por parte de la Dirección General de Recursos Humanos del MINSA.

\section{OFERTA DE ESPECIALISTAS}

Para determinar la oferta, la población objetivo a ser cuantificada y caracterizada está conformada por los médicos especialistas contratados y nombrados de todas las regiones del Perú. Para ello, se recurrió al Sistema de Planillas del MINSA y a la Cartera de Servicios de la Central Nacional de Referencias y Contrarreferencias. El sistema de Planillas reportó información sobre el número de médicos nombrados y contratados, por especialidad y por unidad ejecutora. A partir de este último dato fue factible identificar a qué región pertenecían los profesionales adscritos. La cartera de servicios de los establecimientos de salud de la región Lima proporcionó información sobre el número de especialistas de la Región Lima Metropolitana, Lima Provincias y el Callao. Se confeccionó una matriz de elaboración propia la cual fue remitida a cada Dirección de Salud (DISA) y Dirección Regional de Salud (DIRESA) para consignar la cantidad de médicos especialistas según sexo, rango de edad y tipo de vínculo laboral. Para el caso de cuatro DISA/DIRESA que no enviaron la información solicitada se acudió a la Base de Datos del Sistema de Monitoreo de la dotación de médicos especialistas del MINSA.

\section{DEMANDA DE ESPECIALISTAS}

Para determinar la demanda, se usan dos metodologías de estimación: la primera basada en la demanda de médicos según las normas de categorización y unidades productoras de servicios y la segunda basada en ajustes a la demanda de médicos especialistas según perfil epidemiológico y demográfico local regional. Los datos extraídos para su estimación dependían del tipo de metodología usada.

Para estimar la demanda de médicos, según las normas de categorización y unidades productoras de servicio (UPS), se recurrió a la Norma Técnica 021/MINSA DGSP V 01, denominada "Categoría de Establecimientos del Sector Salud", y a la Información del Portal de transparencia del MINSA, específicamente el Registro Nacional de Establecimientos de Salud y Servicios Médicos de Apoyo (RENAES). Con estas dos fuentes de datos se alcanzó el $100 \%$ de establecimientos de salud categorizados; pero solo fueron considerados los establecimientos de salud categorizados como I-4, II-1. II-2, III-1 y III-2. Adicionalmente, se solicitó a la Dirección General de Salud de las Personas, la relación de establecimientos de salud del MINSA por regiones y según la norma de categorización. Seguidamente, se tomó en cuenta el número de meses por año, días de atención por mes y horas de atención por día de cada tipo de UPS (consulta externa, emergencia, hospitalización y centro quirúrgico) y se estimó el número de médicos especialistas requeridos por tipo de especialidad y por tipo de UPS. A partir de dicha información, se elaboraron matrices que consignaron la necesidad mínima de médicos especialistas que cada establecimiento de salud debería tener según la categoría asignada y las unidades productoras de servicios de salud que deberían ofrecer a la población

Para la estimación de la demanda según el perfil epidemiológico y demográfico, se pretendió inicialmente acceder a la base de datos del Formato HIS de las DISA y DIRESA y a la información sobre perfil epidemiológico y demográfico por regiones contenida en los documentos de análisis de situación de salud de cada una. Sin embargo, estas fuentes no contaban con información completa y actualizada, además que su confiabilidad era dudosa, es por ello que se optó por usar una metodología afín y que ha sido previamente probada en nuestro medio ${ }^{(12)}$. Brevemente, esta metodología utilizó como punto de partida los datos disponibles del Sistema HIS (Health Information System) y de los egresos hospitalarios de la página Web del MINSA. Así, el número de atenciones HIS fueron reducidas a número de atendidos HIS tras considerar las 
categorías de diagnóstico o paciente que sea "nuevo" frente a un "reingreso". Al grupo resultante, se desestimó los atendidos por Medicina General y Odontología. Luego, considerando la fracción de médicos especialistas que deben tener puestos, centros de salud y hospitales, por la norma técnica, se estimó el número de atendidos por especialidades médicas. Finalmente, de acuerdo con la Clasificación internacional de enfermedades, décima versión (CIE-10) se relacionaron los daños o enfermedades con la especialidad correspondiente. Para las especialidades se tuvo en cuenta la nomenclatura de especialidades médicas aprobadas por la Comisión Nacional de Residentado Médico. Adicionalmente, se tomó en cuenta en el cálculo, el número de consultas y su duración (obtenido a partir de consultas a sociedades médico científicas). Para los casos de hospitalización, solo se consideraron las diez primeras causas de cada departamento o región. Con estos parámetros se estimaron tres indicadores

Tabla 1. Distribución de los especialistas médicos, por tipo agregado de especialidad y tipo específico de especialidad, en cuatro subagregados geográficos.

\begin{tabular}{|c|c|c|c|c|c|}
\hline \multirow{2}{*}{ Especialidad } & Nacional & Lima Metropolitana & Callao & Lima Provincias & Resto del Perú \\
\hline & n (\%) & n (\%) & n (\%) & n (\%) & n (\%) \\
\hline \multicolumn{6}{|l|}{ Clinica } \\
\hline Pediatría & $911(24,4)$ & $562(61,7)$ & $56(6,1)$ & $55(6,0)$ & $238(26,1)$ \\
\hline Ginecoobstetricia & $770(20,6)$ & $348(45,2)$ & $45(5,8)$ & $82(10,6)$ & $295(38,3)$ \\
\hline Medicina Interna & $515(13,8)$ & $300(58,3)$ & $30(5,8)$ & $43(8,3)$ & $142(27,6)$ \\
\hline Psiquiatría & $202(5,4)$ & $172(85,1)$ & $5(2,5)$ & $3(1,5)$ & $22(10,9)$ \\
\hline Oncología & $130(3,5)$ & $110(84,6)$ & $4(3,1)$ & $0(0,0)$ & $16(12,3)$ \\
\hline Cardiología & $124(3,3)$ & $69(55,6)$ & $10(8,1)$ & $8(6,5)$ & $37(29,8)$ \\
\hline Neonatología & $121(3,2)$ & $102(84,3)$ & $0(0,0)$ & $6(5,0)$ & $13(10,7)$ \\
\hline Neumología & $121(3,2)$ & $86(71,1)$ & $9(7,4)$ & $8(6,6)$ & $18(14,9)$ \\
\hline Medicina Intensiva & $109(2,9)$ & $75(68,8)$ & $4(3,7)$ & $14(12,8)$ & $16(14,7)$ \\
\hline Gastroenterología & $107(2,9)$ & $54(50,5)$ & $10(9,3)$ & $6(5,6)$ & $37(34,6)$ \\
\hline Emergencias y Desastres & $96(2,6)$ & $82(85,4)$ & $6(6,3)$ & $5(5,2)$ & $3(3,1)$ \\
\hline Medicina Física y Rehabilitación & $77(2,1)$ & $25(32,5)$ & $35(45,5)$ & $3(3,9)$ & $14(18,2)$ \\
\hline Dermatología & $74(2,0)$ & $37(50,0)$ & $7(9,5)$ & $5(6,8)$ & $25(33,8)$ \\
\hline Neurología & $67(1,8)$ & $36(53,7)$ & $9(13,4)$ & $3(4,5)$ & $19(28,4)$ \\
\hline Endocrinología & $59(1,6)$ & $37(62,7)$ & $7(11,9)$ & $4(6,8)$ & $11(18,6)$ \\
\hline Medicina Familiar & $53(1,4)$ & $12(22,6)$ & $2(3,8)$ & $3(5,7)$ & $36(67,9)$ \\
\hline Infectología & $52(1,4)$ & $34(65,4)$ & $5(9,6)$ & $1(1,9)$ & $12(23,1)$ \\
\hline Reumatología & $47(1,3)$ & $29(61,7)$ & $4(8,5)$ & $4(8,5)$ & $10(21,3)$ \\
\hline Nefrología & $46(1,2)$ & $33(71,7)$ & $1(2,2)$ & $2(4,3)$ & $10(21,7)$ \\
\hline Hematología & $25(0,7)$ & $17(68,0)$ & $2(8,0)$ & $1(4,0)$ & $5(20,0)$ \\
\hline Geriatría & $18(0,5)$ & $14(77,8)$ & $0(0,0)$ & $2(11,1)$ & $2(11,1)$ \\
\hline Medicina Legal & $8(0,2)$ & $8(100,0)$ & $0(0,0)$ & $0(0,0)$ & $0(0,0)$ \\
\hline Inmunología & $5(0,1)$ & $5(100,0)$ & $0(0,0)$ & $0(0,0)$ & $0(0,0)$ \\
\hline Genética & $1(0,0)$ & $0(0,0)$ & $0(0,0)$ & $0(0,0)$ & $1(100,0)$ \\
\hline \multicolumn{6}{|l|}{ Quirúrgica } \\
\hline Cirugía General & $650(32,2)$ & $221(34,0)$ & $32(4,9)$ & $68(10,5)$ & $329(50,6)$ \\
\hline Anestesiología & $442(21,9)$ & $219(49,5)$ & $29(6,6)$ & $39(8,8)$ & $155(35,1)$ \\
\hline Traumatología & $254(12,6)$ & $126(49,6)$ & $17(6,7)$ & $26(10,2)$ & $85(33,5)$ \\
\hline Oftalmología & $178(8,8)$ & $101(56,7)$ & $12(6,7)$ & $7(3,9)$ & $58(32,6)$ \\
\hline Otorrinolaringología & $112(5,5)$ & $60(53,6)$ & $7(6,3)$ & $10(8,9)$ & $35(31,3)$ \\
\hline Cirugía Pediátrica & $102(5,1)$ & $67(65,7)$ & $7(6,9)$ & $8(7,8)$ & $20(19,6)$ \\
\hline Neurocirugía & $92(4,6)$ & $58(63,0)$ & $8(8,7)$ & $3(3,3)$ & $23(25,0)$ \\
\hline Urología & $79(3,9)$ & $40(50,6)$ & $8(10,1)$ & $6(7,6)$ & $25(31,6)$ \\
\hline Cirugía Plástica & $54(2,7)$ & $40(74,1)$ & $0(0,0)$ & $3(5,6)$ & $11(20,4)$ \\
\hline Cirugía de Tórax y Cardiovascular & $31(1,5)$ & $25(80,6)$ & $1(3,2)$ & $0(0,0)$ & $5(16,1)$ \\
\hline Cirugía de Cabeza y Cuello & $25(1,2)$ & $21(84,0)$ & $1(4,0)$ & $1(4,0)$ & $2(8,0)$ \\
\hline \multicolumn{6}{|l|}{ Apoyo al diagnóstico } \\
\hline Radiología & $146(49,5)$ & $91(62,3)$ & $13(8,9)$ & $6(4,1)$ & $36(24,7)$ \\
\hline Patología Clínica & $77(26,1)$ & $37(48,1)$ & $1(1,3)$ & $3(3,9)$ & $36(46,8)$ \\
\hline Anatomía Patológica & $72(24,4)$ & $41(56,9)$ & $14(19,4)$ & $3(4,2)$ & $14(19,4)$ \\
\hline \multicolumn{6}{|l|}{ Salud Pública } \\
\hline Gestión/Administración en Salud & $22(100)$ & $8(36,4)$ & $4(18,2)$ & $3(13,6)$ & $7(31,8)$ \\
\hline Total & $6074(100)$ & $3402(56,0)$ & $405(6,7)$ & $444(7,3)$ & $1823(30,0)$ \\
\hline
\end{tabular}


básicos: "Volumen de tiempo en la demanda en consulta externa por especialista en un determinado departamento o región", "Volumen de tiempo en la demanda en hospitalización por especialista en el departamento o región y la "Oferta anual de un especialista en la especialidad" y cuyas aplicaciones han sido descritas previamente ${ }^{(12)}$. Con estos insumos, se estimó el Número de Médicos por especialidad en la Demanda MINSA. Con todas estas consideraciones se alcanzó a estimar las atenciones relacionadas al 99,99\% de enfermedades atendidas por médicos especialistas. El cálculo se repitió para las 25 regiones del país, considerando las 33 especialidades médicas. Se utilizó una matriz de elaboración propia para extraer y sistematizar los datos de estas fuentes.

\section{DETERMINACIÓN DE LA BRECHA}

Para determinar la brecha se estimó la diferencia aritmética simple entre lo estimado en la oferta y lo determinado por cada uno de los dos métodos de estimación de demanda. Esta diferencia se expresa a nivel nacional, por regiones y por tipos de especialidad médica agregada y específica.

\section{RESULTADOS}

\section{OFERTA DE MÉDICOS ESPECIALISTAS}

En el Perú se tuvo una oferta total de 6074 médicos especialistas en el Ministerio de Salud para el año 2010. El 61,5\% (3 738) pertenecen a las especialidades clínicas, el 33,2\% (2 019) a las especialidades quirúrgicas, el 4,9\% (295) a las especialidades de apoyo al diagnóstico y tratamiento y el $0,4 \%$ (22) a las especialidades consideradas por este estudio como de salud pública. Entre las especialidades con mayor oferta de médicos están Pediatría, Gineco-obstetricia, Cirugía, Medicina Interna y Anestesiología, concentrando entre ellas el $54 \%$ de los médicos especialistas. La región Lima Metropolitana concentra al $56 \%$ de los médicos especialistas. Las especialidades Medicina del Deporte, Medicina Ocupacional y del Medio Ambiente no presentan oferta de médicos especialistas según nuestra fuente de información. La información desagregada sobre oferta por tipo genérico y específico de especialidad, así como por agregados regionales y regiones específicas se muestra en la Tabla 1.

\section{DEMANDA DE MÉDICOS ESPECIALISTAS DE ACUERDO A NORMA DE CATEGORIZACIÓN Y UNIDADES PRODUCTORAS DE SERVICIOS DE SALUD (UPS)}

Para el año 2010, se contaba con un total con 7421 establecimientos de salud en el MINSA, de los cuales, 4341 pertenecían a la categoría I-1, 1593 a la categoría I-2, 879 a la categoría I-3, 318 a la categoría I-4, 75 a la categoría II-1, 30 a la categoría II-2, 20 a la categoría III-1 y 10 a la categoría III-2. Se encontraron 155 establecimientos de salud no categorizados.

Para el nivel nacional se encontró una demanda total de 11176 médicos especialistas, de los cuales 7174 (64,2\%) correspondían a las especialidades clínicas, 3131 (28\%) a las especialidades quirúrgicas, $737(6,6 \%)$ a las especialidades de apoyo al diagnóstico y tratamiento y $134(1,2 \%)$ a las especialidades consideradas de gestión y salud pública. Las especialidades con mayor demanda de médicos, según la norma de categorización de establecimientos de salud y Unidades Productoras de Servicios de Salud, son: Ginecoobstetricia con 15\%, Pediatría con 15\%, Cirugía General con $9 \%$ y Medicina Interna con $7 \%$.

\section{DEMANDA DE MÉDICOS ESPECIALISTAS DE ACUERDO AL PERFIL EPIDEMIOLÓGICO Y DEMOGRÁFICO}

La demanda de médicos especialistas según el perfil epidemiológico y demográfico fue de 11738 médicos especialistas, de los cuales 8055 (68,6\%) correspondían a las especialidades clínicas, 3253 $(27,7 \%)$ a las especialidades quirúrgicas, $439(3,7 \%)$ a las especialidades de apoyo al diagnóstico y tratamiento. Las especialidades con mayor demanda de médicos según el Perfil Epidemiológico fueron: Ginecoobstetricia con $17 \%$, Pediatría con $13 \%$, Cirugía General con $12 \%$ y Medicina Interna con $10 \%$.

Para efectos comparativos, se presenta la demanda estimada de acuerdo a las dos metodologías en la Tabla 2.

Tabla 2. Demanda de médicos especialistas a nivel nacional según método usado.

\begin{tabular}{|c|c|c|c|c|}
\hline \multirow{3}{*}{ Tipo de especialista médico } & \multicolumn{4}{|c|}{ Demanda de médicos especialistas } \\
\hline & \multicolumn{2}{|c|}{ Según categorización } & \multicolumn{2}{|c|}{$\begin{array}{c}\text { Según perfil epidemiológico } \\
\text { y demográfico }\end{array}$} \\
\hline & $\mathbf{n}$ & $\%$ & $\mathrm{n}$ & $\%$ \\
\hline Especialidades clínicas & 7174 & $64,2 \%$ & 8055 & $68,6 \%$ \\
\hline Especialidades quirúrgicas & 3131 & $28,0 \%$ & 3253 & $27,7 \%$ \\
\hline Especialidades de apoyo & 737 & $6,6 \%$ & 430 & $3,7 \%$ \\
\hline Especialidades de salud pública & 134 & $1,2 \%$ & 0 & $0,0 \%$ \\
\hline Total & 11176 & $100,0 \%$ & 11738 & $100,0 \%$ \\
\hline
\end{tabular}


Tabla 3. Brechas de médicos especialistas en los establecimientos del Ministerio de Salud del Perú según especialidad agregada y método usado.

\begin{tabular}{|c|c|c|c|c|c|c|c|}
\hline \multirow{2}{*}{ Categoría de especialidades } & \multirow{2}{*}{ Oferta } & \multicolumn{2}{|c|}{ Demanda } & \multicolumn{2}{|c|}{ Brecha 1} & \multicolumn{2}{|c|}{ Brecha 2} \\
\hline & & Método 1 & Método 2 & $\mathbf{n}$ & $(\%)$ & $\mathrm{n}$ & $(\%)$ \\
\hline Especialidades clínicas & 3738 & 7174 & 8055 & -3436 & $(67,3)$ & -4317 & $(76,2)$ \\
\hline Especialidades quirúrgicas & 2019 & 3131 & 3253 & -1112 & $(21,8)$ & -1234 & $(21,8)$ \\
\hline Especialidades de apoyo & 295 & 737 & 430 & -442 & $(8,7)$ & -135 & $(2,4)$ \\
\hline Especialidades de salud pública & 22 & 134 & 0 & -112 & $(2,2)$ & 22 & $(-0,4)$ \\
\hline Total & 6074 & 11176 & 11738 & -5102 & $(100,0)$ & -5664 & $(100,0)$ \\
\hline
\end{tabular}

Oferta: Total de médicos especialistas registrados contratados y nombrados.

Método 1: Por norma de categorización.

Método 2: Por perfil epidemiológico y demográfico

Brecha 1: Diferencia entre oferta y demanda 1

Brecha 2: Diferencia entre oferta y demanda 2

\section{BRECHA ENTRE LA OFERTA Y LA DEMANDA}

La Tabla 3 presenta las brechas agregadas por tipo de especialidad agregada y por método usado.

Al analizar de forma específica por tipo de especialidad, se encuentra que las clásicas cuatro especialidades básicas son las que presentan mayor brecha de médicos especialistas en ambos métodos, agregándosele las especialidades de Medicina Familiar y Anestesiología en el caso del método por norma de categorización. En términos absolutos, por especialidad las brechas se mencionan a continuación. Por el método de Norma de categorización y en orden decreciente: Pediatría = -1067, Ginecoobstetricia = -1 035, Medicina Familiar y Comunitaria $=-618$, Medicina Interna $=-597$, Cirugía general $=-441$ y Anestesiología $=-417$. Por el método de perfil epidemiológico, y en términos absolutos, estas brechas son las siguientes: Ginecoobstetricia $=-1242$, Pediatría $=-758$, Cirugía general $=-747$ y Medicina Interna $=-695$. De donde se aprecia que las especialidades con mayor brecha para ambas metodologías son: Ginecoobstetricia, Pediatría y Cirugía.

En cuanto al análisis de brechas por regiones, y nuevamente en términos absolutos, las cinco regiones con mayores brechas de acuerdo al método basado en la norma de categorización son las siguientes en orden decreciente: Ancash = -643, La Libertad $=-631$, Cusco $=-561$, Lima Metropolitana $=-477$ y Junín $=-473$. Y para el análisis por perfil epidemiológico las cinco mayores brechas a nivel de región son las siguientes Lima Metropolitana $=-1$ 367, La Libertad $=-567$, Lima Provincias $=-426$, Piura $=-413$ y Puno $=-388$. Se aprecia que las regiones con mayor brecha de médicos especialistas para ambas metodologías son: Cusco, La Libertad y Lima Metropolitana.

La evaluación de la brecha en términos relativos, es decir si consideramos el número absoluto de médicos especialistas faltantes respecto al número de médicos especialistas que actualmente existen, arroja por cada una de las regiones estudiadas, los datos reflejados en la Tabla 4, en donde se aprecia que, en contraste con las diferencias absolutas, la región Lima Metropolitana, Lima Provincias y Callao son las que presentan menor déficit relativo, mientras que el mayor se encuentra en Loreto, Piura, Puno y Madre de Dios a partir de las brechas estimadas por ambos métodos.

\section{DISCUSIÓN}

En el presente estudio hemos encontrado que existe una gran brecha entre la oferta y la demanda de médicos especialistas a nivel nacional y regional, que de forma agregada, representa aproximadamente el $45 \%$ del total necesario para cubrir toda la carencia actual, independientemente del método de estimación empleado. De forma consistente, las cuatro especialidades médicas tradicionalmente consideradas como básicas (medicina interna/familiar, pediatría, cirugía general y ginecoobstetricia) son las que exhiben mayor brecha de especialistas, siendo estas variables de acuerdo a la región estudiada. Estos resultados concuerdan con lo registrado en estudios nacionales y extranjeros ${ }^{(6,12,14-17)}$; asimismo, se observa que la brecha de médicos especialistas es mayor en las regiones ubicadas en el quintil 1 y 2 de carencias ${ }^{(18)}$; es decir, los más pobres tienen menor acceso a servicios especializados.

Antes de discutir en extenso los hallazgos de este estudio, algunas de las principales limitaciones de este deben ser mencionadas para brindar elementos de juicio al lector. La principal es que este estudio se basa en fuentes de información secundaria y colección de datos de carácter agregado en muchas ocasiones, por lo que los sesgos de información (específicamente subreporte) 
Tabla 4. Brechas y déficit relativo de médicos especialistas en los establecimientos del MINSA del Perú según método y región.

\begin{tabular}{|c|c|c|c|c|c|}
\hline \multirow{2}{*}{ Región } & \multirow{2}{*}{ Oferta } & \multicolumn{2}{|c|}{ Brechas } & \multicolumn{2}{|c|}{ Déficit relativo } \\
\hline & & Método 1 & Método 2 & Método 1 & Método 2 \\
\hline Loreto & 34 & 285 & 361 & $838,2 \%$ & $1061,8 \%$ \\
\hline Puno & 38 & 396 & 388 & $1042,1 \%$ & $1021,1 \%$ \\
\hline Piura & 49 & 316 & 413 & $644,9 \%$ & $842,9 \%$ \\
\hline Madre de Dios & 12 & 175 & 71 & $1458,3 \%$ & $591,7 \%$ \\
\hline San Martín & 39 & 106 & 213 & $271,8 \%$ & $546,2 \%$ \\
\hline Ayacucho & 50 & 166 & 226 & $332,0 \%$ & $452,0 \%$ \\
\hline Cajamarca & 82 & 314 & 332 & $382,9 \%$ & $404,9 \%$ \\
\hline Ucayali & 43 & 171 & 148 & $397,7 \%$ & $344,2 \%$ \\
\hline Amazonas & 37 & 218 & 124 & $589,2 \%$ & $335,1 \%$ \\
\hline Ancash & 100 & 643 & 299 & $643,0 \%$ & $299,0 \%$ \\
\hline Cusco & 132 & 561 & 391 & $425,0 \%$ & $296,2 \%$ \\
\hline Junín & 79 & 473 & 227 & $598,7 \%$ & $287,3 \%$ \\
\hline Moquegua & 28 & 128 & 71 & $457,1 \%$ & $253,6 \%$ \\
\hline Huánuco & 65 & 118 & 159 & $181,5 \%$ & $244,6 \%$ \\
\hline La Libertad & 235 & 631 & 567 & $268,5 \%$ & $241,3 \%$ \\
\hline Apurimac & 52 & 297 & 106 & $571,2 \%$ & $203,8 \%$ \\
\hline Lambayeque & 86 & 197 & 172 & $229,1 \%$ & $200,0 \%$ \\
\hline Pasco & 25 & 138 & 44 & $552,0 \%$ & $176,0 \%$ \\
\hline Tumbes & 30 & 33 & 52 & $110,0 \%$ & $173,3 \%$ \\
\hline Arequipa & 286 & 307 & 368 & $107,3 \%$ & $128,7 \%$ \\
\hline Huancavelica & 35 & 136 & 43 & $388,6 \%$ & $122,9 \%$ \\
\hline Tacna & 86 & 33 & 88 & $38,4 \%$ & $102,3 \%$ \\
\hline Lima Provincias & 444 & 163 & 426 & $36,7 \%$ & $95,9 \%$ \\
\hline Ica & 200 & 97 & 154 & $48,5 \%$ & $77,0 \%$ \\
\hline Callao & 405 & 124 & 222 & $30,6 \%$ & $54,8 \%$ \\
\hline Lima Metropolitana & 3402 & 477 & 1367 & $14,0 \%$ & $40,2 \%$ \\
\hline
\end{tabular}

Nota: Las brechas son déficits (resta aritmética entra la oferta y la demanda), los déficits relativos son los porcentajes que representan las brechas respecto a la oferta existente.

Método 1: según norma de categorización.

Método 2: según de perfil epidemiológico y demográfico.

deben ser tomados en cuenta. Adicionalmente, la naturaleza agregada de la información disponible debe llamar la atención sobre la verdadera distribución temporal y espacial de las unidades de estudio que eran de interés (en este caso los médicos especialistas cuando se estimaba la oferta). Otras limitaciones que merecen también atención fueron el uso de más de una fuente de datos para la estimación de oferta lo cual debe alertar sobre la diferente rigurosidad con la que se han generado los datos y la posible heterogeneidad introducida en los datos del estudio. De manera similar, los obstáculos administrativos, la falta de datos completos y actualizados o la poca importancia asignada a su generación por parte de las instituciones y autoridades consultadas, debe motivar la reflexión sobre la articulación entre disponer información para evaluación y planificación, contra el acceso transparente, no privilegiado y no exclusivo, esta misma situación se ha presentado en otros países de la región ${ }^{(13,14)}$. Esto último motivó que la metodología de estimación de la demanda por el perfil epidemiológico se base largamente en supuestos teóricos en buena parte.
A pesar de estas limitaciones, los hallazgos de este estudio son importantes pues revelan una realidad poco explorada. Si bien es cierto ya se habían hecho estudios previos sobre la cuantía de médicos y su situación en el contexto de nuestra realidad ${ }^{(10-12)}$, esta no se había centrado en investigar las subespecialidades específicas y las brechas expresadas tanto en forma absoluta como relativa. En estos tiempos en los que los programas de formación académica a nivel de postgrado y pregrado están sujetos a revisión y auditoría constante para velar por sus altos estándares, debería también considerarse la oferta de estas especialidades, no solo de forma más descentralizada o con fines utilitarios, sino de manera más acorde con los requerimientos nacionales y regionales, sin poner en riesgo su calidad educativa y formativa.

Otra notable contribución resta sobre el uso de dos metodologías que, desde diversos frentes y supuestos teóricos, han dado resultados que apuntan en la misma dirección: un déficit grueso y obvio de especialistas el cual debe ser cubierto. Todo indica que no es suficiente utilizar 
una metodología única, ni estándares o parámetros internacionales para la valoración y planificación de recursos humanos en salud ${ }^{(14)}$ y más bien el empleo comparativo de diversas metodologías permite un mejor análisis y enfoque de acciones. El uso conjunto de más de un método en forma paralela, definitivamente va a incrementar la sensibilidad sobre aquello que estamos investigando, aunque el costo puede venir a nivel específico cuando los dos métodos no concuerdan en sus hallazgos como hemos reportado. Por otra parte, aunque los resultados específicos a nivel regional son diferentes, no debemos olvidar que la gran mayoría de resultados presentados se expresan en forma absoluta y ello merece especial consideración al momento de su interpretación. Es por eso que proponemos, además, la valoración mediante el déficit relativo, con la cual se ha obtenido buena concordancia entre lo estimado por los dos métodos.

Además de las consideraciones metodológicas mencionadas, también se pueden mencionar y discutir los hallazgos temáticos clave. Por ejemplo, resalta la carencia de especialistas en salud mental (19) en regiones como Ayacucho que largamente vive los estragos de la violencia política. De igual forma, es notoria la carencia de especialistas dermatólogos en áreas y regiones tropicales si consideramos el especial perfil de morbilidad que estos entornos poseen. Esto debe llamar la atención sobre carencias específicas de cada región no solo en términos cuantitativos, sino, nuevamente, también en función de la realidad social, demográfica y epidemiológica única de cada una.

Similar a lo evidenciado en otro estudio ${ }^{(12)}$, se encontró que más del $50 \%$ de la oferta de médicos especialistas se concentran en Lima Metropolitana, lo cual revela la centralización de los recursos humanos y la inequidad al acceso a los servicios de salud especializados por parte de la población, similares resultados se encontraron en otros estudios de la región ${ }^{(14,15)}$.

Los resultados hallados sobre la demanda según especialidades son similares a los registrados en otros estudios a pesar de emplear otras metodologías ${ }^{(14,16)}$. Estudiar solo la presencia o ausencia de especialistas sería demasiado obtuso puesto que otros factores merecen ser considerados como determinantes o intermediarios. Entre estos podemos citar y proponer los siguientes: la jubilación de los especialistas, los motivos de referencias y contrarreferencias, la práctica de la telemedicina, las migraciones de profesionales dentro y fuera del territorio nacional, las acciones de las estrategias sanitarias nacionales, la conformación de brigadas temporales para satisfacer la provisión de servicios, los incentivos y atractivos económicos y no económicos que afectan la decisión de los especialistas, entre otros factores, los cuales merecen claramente un trabajo más profundo y que escapa a los objetivos del presente estudio.

Finalmente, algunas recomendaciones que se pueden emitir a la luz de los hallazgos son las siguientes: a) Para atender la brecha negativa de médicos especialistas se recomienda, en primera instancia, cubrir la necesidad mínima que el Estado debe proveer a la población, la cual está señalada en diferentes documentos normativos, entre los cuales resalta la norma de Categorización, y b) Sobre la descentralización en la inversión: es importante que las inversiones en oferta de servicios de salud, se den desde o en el marco de la descentralización, para de esta manera, garantizar el acceso equitativo de la población a los servicios de salud, en concordancia con lo establecido en la VII Reunión Regional de los Observatorios de Recursos Humanos en Salud de las Américas que fue realizada en Toronto, Canadá en el $2005^{\left({ }^{(9)}\right.}$.

\section{AGRADECIMIENTOS}

Los autores desean expresar su agradecimiento a todas las personas e instituciones (Direcciones Regionales de Salud, Direcciones de Salud, Dirección General de Gestión del Desarrollo de Recursos Humanos del MINSA) que brindaron información para la elaboración de este trabajo. También agradecen los comentarios y sugerencias de edición por parte de diversos profesionales de la salud que revisaron versiones previas de este manuscrito.

\section{Contribuciones de Autoría}

Todos los autores han participado en la concepción y diseño del trabajo. LZ y BM participaron en la recolección de datos. LZ realizó el análisis e interpretación de datos y en la redacción del manuscrito. BM y RP participaron en la revisión crítica del manuscrito. RP contribuyó adicionalmente en el análisis estadístico. Todos los autores aprobaron la versión final del documento.

\section{Fuentes de Financiamiento}

Dirección General de Gestión del Desarrollo de los Recursos Humanos, Ministerio de Salud, Lima, Perú.

\section{Conflictos de Interés}

Los autores declaran no tener conflictos de interés en la publicación de este artículo. 


\section{REFERENCIAS BIBLIOGRÁFICAS}

1. Ministerio de Salud del Perú. Informe Técnico Final de la Comisión Multisectorial encargada de proponer los mecanismos necesarios que permitan consolidar un Sistema Nacional de Salud. Lima: MINSA; 2009.

2. Ministerio de Salud del Perú. Cuentas Nacionales de Salud, Perú 1995-2005, Lima: MINSA/ CIES; 2008.

3. Instituto Nacional de Estadística e Informática. Condiciones de vida en el Perú. Lima: INEI; 2011.

4. Ministerio de Salud del Perú. Análisis de Situación de Salud del Perú. Lima: MINSA; 2010.

5. Ministerio de Salud del Perú. Lineamientos de Gestión del Ministro de Salud. Lima: MINSA; 2008.

6. Observatorio Nacional de Recursos Humanos en Salud del Perú (IDREH). El Perú respondiendo a los desafíos de la década de los recursos humanos en salud. Lima: MINSA; 2006.

7. Instituto de Desarrollo de Recursos Humanos. Formación y Regulación de los Recursos Humanos en Salud. Lima: MINSA; 2005.

8. Observatorio de Recursos Humanos en Salud. Propuesta Interagencial OPS-CEPAL-OIT. Quito: OPS/OMS; 2000.

9. Organización Panamericana de la Salud. Informe de la VII Reunión Regional de los Observatorios de Recursos Humanos en Salud: Hacia una década de Recursos Humanos en Salud para las Américas. Toronto: OPS; 2005.

10. Madueño M, Alarcon J, Sanabria C. Análisis de la brecha entre la oferta y la demanda de servicios de salud para la programación de la inversión sectorial de mediano plazo. Lima: PHRplus Perú; 2003.

11. Baanante M, Escalante G, Pollarolo P. Informe Final: Estudio sobre los recursos humanos en salud en el Perú: Gestión, distribución, normatividad, regulación, condiciones laborales, salariales y formación. Lima: GRADE; 2006.
12. Colegio Médico del Perú. Análisis actual y prospectivo de la oferta, demanda y necesidad de médicos en el Perú 2005-2011. Lima: CMP; 2007.

13. Barria S, Contardo N, Caravantes R, Monasterio H. Manual de análisis de la dotación de personal en establecimientos de salud. Brasilia: OPS; 2004.

14. Subsecretaria de Redes Asistenciales. Estudios de Brechas de oferta y demanda de médicos especialistas en Chile. Santiago de Chile: MINSAL; 2010.

15. Rosselli D. Otero A. Heller D. Calderón C. Moreno S, Pérez A. Estimación de la oferta de médicos especialistas en Colombia con el método de captura-recaptura. Rev Panam Salud Publica. 2011;9(6):393-8.

16. López-Valcárcel BG, Pérez BP. Oferta y Necesidad de Médicos Especialistas en España 2006 - 2030. Grupo de Investigación en Economía de la Salud. Las Palmas de Gran Canaria: Universidad de Las Palmas de Gran Canaria; 2007.

17. Barber P, González B. Oferta y Necesidad de Médicos Especialistas en España (2008-2025). Madrid: Ministerio de Sanidad y Consumo; 2009.

18. Fondo de Cooperación para el Desarrollo Social. Mapa de Pobreza 2006. Lima: FONCODES; 2006.

19. Defensoría del Pueblo. Informe Defensorial № 140 : Salud mental y Derechos Humanos, Supervisión de la política pública, la calidad de los servicios y la atención a poblaciones vulnerables. Lima: Defensoría del Pueblo; 2009.

Correspondencia: Leslie Zevallos

Dirección: Prolongación Húsares de Junín N. ${ }^{\circ} 116-$ Urb. Retablo -Comas, Lima 7, Perú.

Teléfono: (511) 250-5056 / (511) 993-028543

Correo electrónico: Iczq2002@yahoo.es

\section{Consulte la versión electrónica de la Revista Peruana de Medicina Experimental y Salud Pública en} www.pubmed.gov

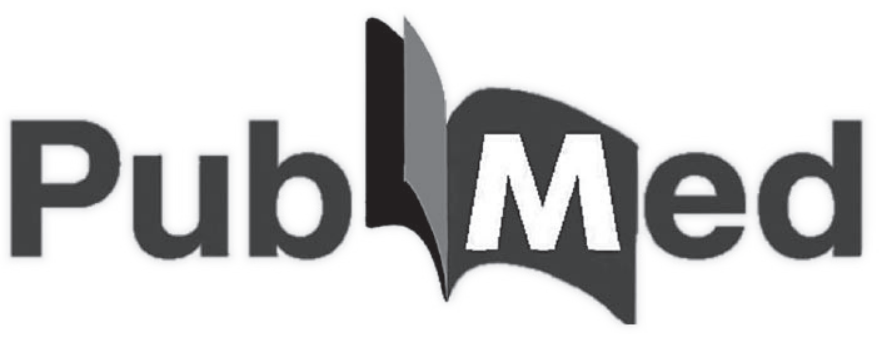

